\title{
Labyrinthe
}

38 | 2012 (1)

L'éloquence des singes

\section{Essai de sémantique simiesque}

Apes and Monkeys. Their Life and Language

\section{Richard Lynch Garner}

Traducteur : Grégoire Leménager et Laurence Marie

\section{CpenEdition}

Journals

\section{Édition électronique}

URL : http://journals.openedition.org/labyrinthe/4240

DOI : $10.4000 /$ labyrinthe.4240

ISSN : 1950-6031

Éditeur

Hermann

Édition imprimée

Date de publication : 1 avril 2012

Pagination : 11-25

ISBN : 9782705682361

Référence électronique

Richard Lynch Garner, «Essai de sémantique simiesque », Labyrinthe [En ligne], 38 | 2012 (1), mis en ligne le 01 avril 2014, consulté le 01 mai 2019. URL : http://journals.openedition.org/labyrinthe/4240 ; DOI : $10.4000 /$ labyrinthe.4240

Propriété intellectuelle 


\title{
Essai de sémantique simiesque
}

\author{
Richard Lynch GARNER
}

Richard Lynch Garner (1848-1920), Américain à la fois naturaliste, chasseur, explorateur, est l'auteur de plusieurs ouvrages sur les systèmes de communication vocale parmi les primates, où figurent Apes and Monkeys, dont nous traduisons ici des extraits en français pour la première fois. Garner ne fut pas un grand savant, mais, en posant l'hypothèse de ce qu'il nommait une «langue simiesque », il chercha à isoler des sons signifiants émis par les singes, à les noter, et les tester. Garner fut en effet le premier à éprouver la possible portée sémantique d'appels réalisés par des singes en enregistrant ces vocalisations avec un phonographe et en les jouant à d'autres animaux, en vue d'établir la compréhension du signal. Par sa démarche, plus que par ses résultats, Garner est un pionnier, préfigurant des expériences commencées dans les années 1970, et qui s'attachent à identifier des «mots", voire une syntaxe, parmi les cris que profèrent les singes dans leur milieu naturel ${ }^{1}$. Il est également le contemporain d'essais en psychologie, qui, repartant d'idées anciennes, essaieront d'enseigner le langage humain à des grands singes. Ces deux types d'enquêtes forment le cœur du présent dossier.

L. D.

Texte original: Apes and Monkeys. Their Life and Language. Boston \& Londres, Ginn \& Company, 1900, p. 12-23 et 108-116.

1. Cf., dans les « Paroles de singes », à la fin de ce dossier, les extraits [6], [13], [49]. Pour des compléments historiques, lire Gregory Radick, The Simian Tongue, Chicago, The University of Chicago Press, 2007. 


\section{Labyrinthe, $n^{\circ} 38$}

J'ai grandi au milieu des collines bleues et des lacs cristallins des Appalaches, loin de l'artifice des grandes métropoles : je vivais dans des conditions plus primitives, moins compliquées que dans l'effervescence des zones très peuplées. La nature fut mon premier professeur et les animaux domestiques mes premiers compagnons. Ma jeunesse s'écoula dans ce cadre et c'est là que je conçus pour la première fois l'idée que les animaux parlent. Enfant, je croyais que tous les animaux d'une même espèce pouvaient se comprendre. Et je me rappelle de nombreuses circonstances où ce fut clairement le cas.

Les adultes disaient que les animaux savaient communiquer entre eux, mais leur déniaient la capacité de parler. Or, le petit garçon que j'étais s'accrochait fermement à l'idée que les sons émis par les animaux constituaient une parole. Je me pose d'ailleurs encore la question aujourd'hui : en quoi ne constituent-ils pas une parole? Cette question en appelle une autre.

Qu'est-ce que la parole ? C'est un son produit oralement et volontairement en vue de transmettre, de l'esprit de l'orateur vers l'esprit d'un destinataire, une idée qui a été conçue en amont. Un son oral qui a été élaboré de cette manière et qui remplit cette fonction dans le système animal relève de la parole.

Certes, quand on les compare à ceux des hommes, les vocabulaires utilisés par les animaux apparaissent très limités. Mais leur existence n'en est pas moins réelle. Certes, une idée se forme avec probablement moins de netteté dans l'esprit d'un animal que dans l'esprit d'un être humain. Mais la même idée n'apparaît pas toujours avec la même clarté dans l'esprit de deux êtres humains différents. Son imprécision n'enlève rien à sa réalité.

L'expression est la forme matérialisée de la pensée, et la parole un mode d'expression. Chaque animal est capable d'exprimer une pensée qu'il a su concevoir, et cette expression est aussi claire que la pensée exprimée. Supposer qu'une créature peut être douée de la faculté de penser, mais pas du moyen de l'exprimer est incompatible avec quelque conception de la nature que ce soit.

Il est vrai que certains sons vocaux exprimant l'émotion, comme la douleur ou le plaisir, ne peuvent pas, à proprement parler, être considérés comme de la parole, bien qu'il soit possible d'en déduire l'état d'esprit 


\section{Essai de sémantique simiesque}

correspondant. Mais même s'ils ne constituent pas réellement une parole, ils apparaissent comme la matrice à partir de laquelle elle se développe. Même si les émotions ne sont pas volontaires, elles n'existent pas en dehors de l'esprit. Elles sont produites par des causes externes, et la ligne de démarcation qui les sépare de formes de pensées plus définies est imprécise et mouvante. La pensée peut bien être involontaire, mais l'expression naît du désir, et c'est l'unique motivation de la parole.

Ce travail n'a pas pour objectif de discuter de questions spécifiquement psychologiques. Les avancées de la psychologie seront mobilisées uniquement comme des arguments sur lesquels nous nous appuierons pour montrer que les animaux possèdent la faculté de parler. Cette étude a consisté à collecter des faits observés - les psychologues pourront en tirer leurs propres conclusions.

J'étais habité par le sentiment tenace que les animaux se parlent entre eux. D'année en année, j'observai un certain nombre de faits tendant à le confirmer. Mais mes doutes et mes hésitations furent définitivement balayés après un événement qui se produisit il y a environ seize ans. Avant cette révélation, j'avais observé que les animaux appartenant aux espèces les plus développées semblaient posséder les types de langage les plus élaborés. Et au fur et à mesure de mes observations, je m'étais attaché à classer de nombreux faits au sein d'un tableau. En 1884, je me rendis au Jardin zoologique de Cincinnati, où le comportement d'une bande de singes qui partageaient leur cage avec un grand mandrill éveilla mon intérêt.

Il apparaissait clairement que ce babouin sauvage suscitait la terreur chez les plus petits pensionnaires de la cage. Un mur de brique séparait la cage en deux compartiments. L'un était prévu pour l'été et l'autre pour l'hiver. Dans ce mur, s'ouvrait une petite porte assez large pour laisser passer les occupants. Je remarquai que deux ou trois singes surveillaient en permanence le comportement du babouin et informaient leurs congénères de chacun de ses mouvements.

Quand le babouin reposait calmement, les singes allaient et venaient sans crainte. Mais dès qu'il se dressait sur ses pieds ou montrait un signe d'inquiétude, les surveillants en informaient aussitôt ceux qui se trouvaient dans l'autre compartiment, et ceux-ci agissaient conformément à l'alerte. Je ne parvenais pas à déterminer le contenu exact de l'information transmise, mais sa nature était évidente. 
Je décidai alors d'en apprendre davantage sur sa signification. Après avoir passé des heures à observer le comportement des singes et à écouter les sons qui le déterminaient, je parvins à la certitude que ce que disaient les surveillants était suffisamment défini pour guider les actions de ceux auxquels ils s'adressaient. J'aurais même volontiers accepté de me fier à ces avertissements pour assurer ma propre sécurité. Après avoir brièvement étudié ces sons, j'étais capable de comprendre comment se comportait le babouin avec ses voisins; et même si son message ne contenait aucun détail élaboré que j' aurais pu comprendre, la nature de son comportement me paraissait évidente. J'observai qu'un signal sonore particulier conduisait les singes à se comporter d'une certaine manière, et tel autre son d'une autre manière.

À partir de ce moment-là, je décidai d'apprendre le langage des singes. Je ne soupçonnais alors pas l'ampleur de la tâche et je n'avais pas anticipé les difficultés qui se sont manifestées par la suite. D'année en année, avec les nouvelles idées qui me venaient surgissaient de nouvelles barrières. L'horizon à découvrir devenait sans cesse plus vaste. Malgré tout, je ne me décourageai pas des piètres résultats produits par mes premiers efforts. De temps à autre, j'allais voir les différents groupes de singes en captivité de ce pays, et je saisissais même toutes les occasions d'observer ceux qui accompagnaient les spectacles itinérants, les joueurs d'accordéon, etc.

Après des années d'études sans protocole défini, je me dis que le phonographe pourrait m'aider efficacement à résoudre le problème. Il me permettrait en effet de comparer de façon plus précise les sons émis par les différents singes. Après avoir dûment considéré la chose, je me rendis à Washington pour faire part de mon idée au docteur Baker, de la Smithsonian Institution. Le docteur commença par sourire, mais quand je lui eus expliqué la manière dont je comptais parvenir à mes fins, il jugea que cette trouvaille inédite pourrait faire avancer la science du langage.

M'étant procuré un phonographe, je me retirai dans la maison des animaux qui jouxtait alors la Smithsonian Institution. À l'époque, s'y trouvaient deux singes, qui constituaient le noyau autour duquel s'est développé depuis l'actuel Parc zoologique national de Washington. Ces deux singes n'étaient pas de la même espèce, mais ils avaient un temps occupé la même cage. Je fis déménager la femelle de sa cage pour l'installer dans une autre. Je plaçai le phonographe près de sa cage et la femelle fut incitée par divers moyens à proférer quelques sons, qui furent 
enregistrés sur le cylindre de cire. L'appareil fut ensuite placé près de la cage du mâle et on lui fit entendre l'enregistrement. Son comportement montrait clairement qu'il reconnaissait les sons et comprenait leur nature. Il scrutait le pavillon d'où sortaient les sons et paraissait perplexe de ne pas y voir le singe qui les avait émis. Il cherchait la source correspondant au son et, ne parvenant pas à trouver sa camarade, il enfonça brusquement son bras dans le pavillon du phonographe. Il en palpa les bords dans le vain espoir de la trouver. L'expression de son visage formait une étude digne des meilleurs efforts des physiognomonistes.

Ensuite, quelques sons de sa voix furent enregistrés sur un autre cylindre et diffusés à la femelle, qui montra des signes de reconnaissance; mais comme cet enregistrement n'était pas très distinct, il ne suscita pas chez elle l'intérêt que l'autre enregistrement avait provoqué chez le mâle.

Sans le moindre doute, ce fut, dans toute l'histoire du langage, la première tentative de consigner le langage des singes sous forme d'enregistrement sonore. En dépit du caractère rudimentaire de cette première expérience et du caractère peu concluant de ses résultats, elle indiquait la voie et engageait à poursuivre les efforts pour trouver la source d'où coule la grande rivière du langage humain.

Des critiques s'élevèrent à l'époque pour affirmer que cette expérience ne pouvait avoir de valeur scientifique parce que le singe avait été incité à émettre les sons enregistrés et que le résultat comportait uniquement des sons de colère ou des jurons. Que ce soit des mots convenables ou des jurons n'était pas mon problème, pourvu qu'il s'agisse de sons proférés par un singe et qu'ils soient reconnus comme tels par d'autres singes: $\mathrm{si}$ un singe utilise des jurons, c'est qu'il possède sans aucun doute d'autres formes de langage.

Peu de temps après cette expérience, je me rendis à Chicago, où j'enregistrai un capucin brun. Je choisis un son très communément utilisé par cette espèce. Je ne connaissais pas sa signification exacte, mais la fréquence de son usage m'incitait à le considérer comme l'un des mots les plus importants. Ayant obtenu l'enregistrement souhaité, je rentrai à New York. J'y choisis un singe de la même espèce et lui diffusai l'enregistrement. Le singe donna immédiatement des signes de compréhension et se mit à lui répondre. Je répétai le même son encore et encore, et le singe lui répondait à chaque fois. Il regardait le pavillon d'où sortait le son, puis les mouvements de l'appareil, et se reculait. Mais comme le son continuait à sortir du pavillon, son intérêt parut piqué au 
vif. S'approchant du pavillon, il jeta un coup d'œil prudent à l'intérieur. Le son se fit entendre une nouvelle fois. Il enfonça son bras dans le pavillon et regarda alentour pour voir si, en effrayant l'autre singe, il ne l'avait pas conduit à s'enfuir. Comme il n'arrivait pas à le trouver, il ôta à nouveau son bras du pavillon, puis répondit aux sons. Il semblait considérer la chose avec une forme de superstition et avoir conscience qu'un singe aurait dû être présent. Ne parvenant pas à le trouver, il prit un air soupçonneux. Je ne sais pas dans quelle mesure il considérait ce phénomène comme surnaturel, mais il avait évidemment conscience de son étrangeté.

Dans cette expérience, plusieurs faits méritent d'être notés. L'enregistrement n'a transmis au singe que le son froid, mécanique. Les éléments para-verbaux, comme le geste, ont été entièrement éliminés et ne sont donc pas intervenus. Le singe n'avait dès lors que le son à interpréter, rien d'autre. Voilà qui tendrait à montrer que le langage sonore du singe, de même que celui de l'homme, porte en lui une signification fixe et constante. Cette conclusion a été confirmée depuis par des expériences nombreuses et variées, réalisées avec des procédés mécaniques de différentes sortes.

Un des défauts de cette expérience, c'est que je n'avais pas prévu le moyen d'enregistrer le son émis en réponse à celui du phonographe. Je me procurai donc un autre appareil pour y remédier. Ayant ainsi pu enregistrer une réponse, je disposais de deux cylindres pour comparer les sons. Je répétai l'expérience de la même manière : je diffusai l'enregistrement avec un appareil et enregistrai la réponse avec l'autre, jusqu'à avoir obtenu des enregistrements des sons qui composent la langue de presque tous les singes en captivité dans ce pays. Je réalisais ces prises de son pendant mon temps libre, je les comparais et les étudiais attentivement, jusqu'à être capable d'interpréter neuf sons du langage des singes capucins, et, incidemment, quelques sons d'un grand nombre d'autres espèces.

Il est absolument impossible de rendre compte littéralement des sons du langage simiesque. Il est également difficile de leur trouver un équivalent exact dans le langage humain. Mais pour donner une idée de la nature et de l'étendue du langage des singes, je veux tout de même tenter de décrire un mot ou deux. Dans la langue du capucin brun, le mot le plus important se prononce [huw], avec un [u] long. L'effet phonétique est riche et musical. L'élément vocalique qui domine est un [u] purement vocal. La signification de base de ce son renvoie à la nourriture, qui est 
au centre des pensées de tous les singes. Le son ne désigne pas seulement la nourriture au sens concret, c'est-à-dire les aliments comestibles, mais il renvoie parfois aussi à l'acte de manger et il comporte dans ce sens les caractéristiques d'un verbe. À d'autres moments, il renvoie au désir de manger ou à la sensation de faim, et dans ce cas on peut dire qu'il revêt les caractéristiques d'un adjectif. Toutefois, comme les valeurs grammaticales dépendent de structures et que le langage des singes est monophrastique, on ne peut vraiment parler de forme grammaticale à son endroit. Pour ce que j'ai pu observer, tous les sons proférés par ces espèces sont des monosyllabes; et ils ne contiennent pour la plupart qu'un seul élément phonétique distinct. C'est pourquoi je les ai décrits comme étant «monophonétiques ». Le mot prononcé [huw] est parfois utilisé avec la volonté apparente d'exprimer l'amitié, ou quelque chose de ce genre.

Un autre mot qui renvoie au fait de boire, ou au liquide, commence avec un [x] faiblement guttural, qui se prolonge par un son ressemblant à la diphtongue $[\varnothing]$ et se termine par un [j] s'évanouissant progressivement. Le son est utilisé en relation avec la boisson, à peu près de la même manière que le son [huw] pour la nourriture.

Je n'ai jusqu'ici pas trouvé trace des voyelles longues [a], [e], [i] ou [o]. Mais j'ai repéré un élément vocalique ressemblant à un [i] bref dans un cri d'alerte émis sous l'effet du stress causé par une grande frayeur ou la menace d'une agression. Ce son est proféré à une hauteur d'environ deux octaves au-dessus d'une voix de femme.

Tous les sons émis par les singes et, pour ce que j'en ai observé, par les autres animaux, renvoient à leurs besoins physiques naturels. Les singes ne sont pas capables d'exprimer des pensées complexes ou des abstractions parce que les animaux n'ont pas ce type de pensées. La simplicité de leurs modes de vie n'exige pas de pensées complexes.

Un mot que « Nellie » (l'un de mes singes domestiques) utilisait pour me prévenir d'un danger me fit apercevoir un point commun frappant entre les langages humain et simiesque. Il ne s'agit pas du son que j'ai décrit plus haut comme le cri d'alerte utilisé en cas de danger imminent. Le son dont il est question ici est émis en cas de danger lointain ou pour annoncer quelque chose d'inhabituel. Dans une transcription aussi peu approximative que possible, cela donne quelque chose comme [isidzike]. Mes petits amis m'ont souvent donné l'alerte à l'aide de ce mot. 
La cage de Nellie se trouve à côté de mon bureau. La nuit, elle voulait sans cesse rester éveillée aussi longtemps que la lumière était allumée. J'ai toujours veillé jusqu'à des heures tardives et il n'était pas question que je bouleverse mes habitudes pour lui offrir une bonne nuit de sommeil. Un jour, vers deux heures du matin, alors que j'étais sur le point de me retirer, je trouvai Nellie complètement éveillée. Je tirai une chaise près de sa cage et m'assis pour la regarder se livrer à ses clowneries. Elle essayait de me divertir à l'aide de clochettes et de jouets. Sans lui laisser voir ce que je faisais, j'attachai un long fil à un gant que je plaçai dans un coin de la pièce, à quelques mètres d'elle. En tirant sur la ficelle, je fis avancer le gant sur le sol à travers la pièce. La première fois que je tendis la ficelle, qui passait par-dessus l'un de mes genoux et en dessous de l'autre, le gant bougea de manière quasi imperceptible. Son œil vif s'en aperçut immédiatement. Se tenant presque sur la pointe des pieds, la bouche entrouverte, elle scrutait attentivement le gant. Puis, à voix basse, presque en chuchotant, elle émit le son [isidzikz]. Elle le répéta à peu près toutes les secondes, tout en cherchant à voir si j'étais conscient ou non de la présence de ce lutin qui approchait. Son comportement était très proche de celui d'un homme. Ses mouvements étaient aussi furtifs que ceux d'un chat. À mesure que le gant se rapprochait, elle devenait de plus en plus démonstrative. Et quand elle vit le monstre grimper sur la jambe de mon pantalon, elle émit le son d'une voix grave et très rapidement.

Elle tenta d'atteindre l'objet. Elle pensait évidemment qu'il était vivant. Elle repéra le fil qui avait servi à tirer le gant à travers la pièce, mais elle paraissait douter du fait qu'il avait pu jouer un rôle. Elle suivit plusieurs fois le fil des yeux, de mon genou jusqu'au gant, mais je ne pense pas qu'elle ait découvert ce qui faisait bouger le gant. Après avoir renouvelé l'expérience à plusieurs reprises, avec à peu près le même résultat à chaque fois, je soulageai son inquiétude en lui permettant d'examiner le gant. Elle le fit avec un intérêt soutenu pendant un moment, puis s'en détourna. Je retentai la même manœuvre, mais je ne parvins plus à susciter chez elle le moindre intérêt après qu'elle eut examiné le gant.

Quand Nellie avait vu pour la première fois le gant bouger sur le sol, elle avait tenté d'attirer mon attention à voix basse. À mesure que l'objet approchait, elle avait pris la chose de plus en plus au sérieux et émis le même son un peu plus fort. Quand elle avait découvert que le monstre (ainsi considérait-elle le gant) grimpait sur ma jambe, elle avait émis le signal d'alerte d'une voix suffisamment forte pour franchir la distance 
qui me séparait d'elle. Ces faits indiquent que sa perception du son était très précise. Elle avait bien l'intention de m'avertir de l'approche d'un danger, mais sans que l'élément menaçant ne s'en rende compte. Son signal était devenu plus pressant à mesure que le danger augmentait. Quand elle vit qu'il était imminent, elle cessa de dissimuler ou de contenir son inquiétude.

Nellie était une petite créature très affectueuse. Elle détestait qu'on la laisse seule, même avec une abondante provision de jouets et de nourriture. Quand elle me voyait enfiler mon pardessus ou attraper mon chapeau, elle pressentait qu'elle allait se retrouver seule. Elle se mettait alors à supplier, à quémander, à jacasser. Je le regardais souvent à travers un petit trou percé dans la porte. Quand elle était tout à fait seule, elle s'amusait avec ses jouets dans un parfait silence. Il arrivait qu'elle ne dise pas un mot pendant des heures entières. Elle ne faisait pas exception à la règle selon laquelle les singes ne parlent pas quand ils sont seuls.

Même si le langage des singes est inférieur à celui des hommes, il est d'une éloquence qui apaise et ce qu'il exprime touche l'homme au cœur.

En bref, le langage des singes et celui des hommes se ressemblent sur tous les points essentiels. Les sons qui composent le langage des singes sont volontaires, intentionnels et articulés. Ils s'adressent aux autres clairement dans l'objectif d'être compris. Celui qui parle montre qu'il sait quel message il souhaite transmettre par la médiation du langage. Il attend et il s'attend à une réponse. Si celle-ci ne vient pas, il répète le son. Celui qui parle regarde en général celui auquel il s'adresse. Les singes ne prononcent généralement pas ces sons quand ils sont seuls. Ils comprennent les sons émis par d'autres singes appartenant à la même espèce qu'eux. Ils comprennent aussi les sons quand ils sont imités par un être humain, un phonographe ou d'autres moyens mécaniques. Ils comprennent les sons sans que ceux-ci soient accompagnés de signes ou de gestes. Ils interprètent le même son toujours de la même manière. Ils produisent les sons à l'aide de leurs cordes vocales et les modulent avec leurs dents, leur langue, leur palais et leurs lèvres. Leur langage comporte des nuances dialectales et les animaux les plus évolués ont des types de langage plus développés que ceux qui le sont moins. Les types de langage les plus développés sont légèrement plus complexes et ils ont une signification un peu plus précise que les formes inférieures. Dans son état actuel, le langage des singes semble résulter du développement des formes inférieures de langage. Chaque race ou espèce de singes a une 


\section{Labyrinthe, $n^{\circ} 38$}

forme de langage qui lui est propre. Quand ils sont réunis dans une même cage pendant un certain temps, ils apprennent ce que signifient les sons des autres, mais ils essaient rarement de les prononcer. Leur aptitude au langage est proportionnelle à leurs capacités mentales et à leur position sociale au sein du groupe. Ils émettent des sons plus ou moins forts en fonction des circonstances, ce qui indique qu'ils connaissent les valeurs attachées au son. Plus les règles de vie collective sont marquées chez une espèce, plus le type de langage utilisé est élaboré. Pour autant que je puisse en juger, il n'existe pas de différence intrinsèque entre le langage des singes et le langage des hommes.

\section{II}

Le langage des chimpanzés (comme celui des autres singes) se limite à un petit nombre de sons, qui renvoient principalement à leurs besoins naturels. Pris dans sa totalité, leur vocabulaire n'excède probablement pas vingt-cinq à trente mots. La plupart d'entre eux sont vagues ou ambigus, mais ils expriment ce que pense le singe avec autant de précision qu'il y en a dans son esprit, et assez distinctement pour qu'il dise ce qu'il veut dire.

Pendant mes recherches, j'ai appris dix mots du langage des chimpanzés, de sorte que je peux les comprendre et me faire comprendre d'eux. Pour ce qui est du ton, du volume, et des modulations, la plupart des sons ont la même amplitude que la voix humaine. Deux d'entre eux sont beaucoup plus puissants, en volume sonore, que ce que des poumons humains permettent d'atteindre, et l'un d'eux s'élève dans les aigus plus d'une octave au-dessus d'une voix humaine ordinaire. Ces deux sons sont audibles à une grande distance, mais ils ne sont pas véritablement inclus dans les limites du langage.

Il y a entre les organes de la voix chez le chimpanzé et chez l'homme autant de ressemblance que pour ce qui concerne leurs autres caractéristiques physiques. Ils diffèrent toutefois légèrement sur un point qui mérite d'être signalé. Juste au-dessus de la cavité qu'on appelle la glotte et qui est située entre les cordes vocales, se trouvent deux petits sacs ou ventricules. Ils sont plus larges et plus souples chez le singe que chez l'homme. Lorsque l'on parle, ils sont gonflés par l'air qui vient des poumons à travers le long tube appelé le larynx. La fonction de ces ventricules est de contrôler 
et de modifier le son en augmentant ou en diminuant la pression de l'air envoyé dans le tube. Ils servent en même temps de réservoir et de jauge.

Lorsque les chimpanzés émettent leurs sons les plus puissants, ces ventricules se dilatent beaucoup. Cela renforce la voix ou augmente son volume. C'est en partie grâce à ces petits sacs que le singe est capable d'émettre un cri si puissant et si perçant. Mais la tonalité et le volume de sa voix ne viennent pas uniquement de là, car le gorille (chez qui ces ventricules sont beaucoup plus petits) peut émettre un son large et puissant. Il est toutefois possible que nous fassions erreur à propos du son associé au gorille.

Bien que les sons produits par le chimpanzé soient imitables par la voix humaine, aucun système de symboles phonétiques en vigueur parmi les hommes ne peut les exprimer ou les représenter. Les alphabets sont issus des pictogrammes et le symbole conventionnel utilisé pour représenter un son donné n'a pas de rapport avec les organes de la parole qui le produisent. Les quelques lignes figées qui sont parvenues jusqu'à nous et qui forment désormais les alphabets sont en elles-mêmes dépourvues de signification. Mais elles ont si longtemps servi à représenter les sons élémentaires du langage qu'il serait difficile de les remplacer par d'autres.

Comme aucune transcription littérale ne permet de représenter les éléments phonétiques du langage des chimpanzés, j'ai franchi une nouvelle étape dans l'art de l'écriture. Je suggère un système de symboles qui est rationnel dans sa méthode et simple à utiliser.

Les organes de la parole s'actionnent toujours en harmonie les uns avec les autres. Tel mouvement des lèvres est toujours accompagné par tel mouvement des organes internes de la parole. C'est vrai chez le singe comme chez l'homme. Pour prononcer le même son, chacun se sert des mêmes organes, et de la même façon.

C'est par ces moyens que les sourds-muets sont capables de distinguer les sons du langage et de les reproduire sans les entendre. Après un long travail d'apprentissage, ils parviennent à distinguer jusqu'aux nuances sonores les plus subtiles.

Cette évidence donne la clé de la méthode que je propose. Cette méthode en est pour l'instant à un stade peu avancé, mais je crois possible de réussir à ce qu'elle représente, à l'aide d'un très petit nombre de symboles, toute la série des sons vocaliques émis par l'homme ou les autres animaux.

Les principaux symboles que j'utilise sont les parenthèses qu'on trouve communément dans l'imprimerie. Les deux traits incurvés dont les côtés convexes sont opposés, comme cela ( ), représentent la glotte ouverte, 
dans la position qui permet à la voix de prononcer le son très ouvert du [a], comme dans « pâte ». La glotte à moitié fermée prononce le son [o]. Pour transcrire ce son, il faut insérer un point entre deux traits incurvés, comme cela (.). Quand l'aperture est encore plus resserrée, cela produit le son [u], comme dans « loup ». Pour représenter ce son, il faut insérer deux points entre les parenthèses, comme cela (:). Quand l'aperture se réduit encore, cela donne le son $[\varnothing]$, comme dans « deux ». Une apostrophe entre les deux parenthèses permet de transcrire ce son, comme cela ('). Quand les cordes vocales subissent une forte tension, et que l'orifice est presque fermé, cela donne le son $[\varepsilon]$, comme dans « fête ». On transcrit ce son en plaçant un tiret entre les deux parenthèses, comme cela (-). Il s'agit là des principaux sons vocaliques utilisés par tous les animaux, et qui sont parfois modifiés chez l'homme. Chez l'homme, vient s'ajouter le son du [ø] long, qui comme le [o] long est rarement utilisé par le singe.

Ces voyelles élémentaires sont la base de tous les autres sons, et c'est avec des signes diacritiques indiquant les mouvements accomplis par les organes de la parole que l'on peut transcrire les éléments consonantiques.

Une seule parenthèse, avec le côté concave tourné vers la gauche, représentera le son [w] à l'initiale, que l'on entend parfois chez les animaux. On peut l'utiliser en le plaçant à la gauche du symbole principal, comme cela ) ( ), et ce symbole, en tant que tel, se prononce presque comme [wa], le [w] étant élidé au point d'être presque inaudible. Si l'on oriente le côté concave vers la droite, en le situant à droite du symbole principal, comme cela ( ) (, cela représente le son [w] s'évanouissant en fin de mot. Ce symbole se lit [aw], la voyelle initiale étant absorbée par le son final [w]. Une apostrophe placée avant ou après le symbole représente [f] ou [v]. L'accent grave (comme dans « è »), placé avant ou après le symbole, représente le son du $[\mathrm{h}]$ expiré; et l'accent aigu, comme dans « é », indique un son aspiré.

Accompagner le symbole d'un numéro en exposant permet d'indiquer la hauteur du son. Si le symbole ne comporte pas d'exposant, le son est tel que l'émettrait une voix humaine dans un contexte ordinaire. La lettre « $\mathrm{X}$ » indique que le son est répété, et le chiffre qui la suit donne le nombre de répétitions (et non sa hauteur). Par exemple, nous écrirons (.) pour le [o] long prononcé dans une tonalité normale; le même symbole noté $(.)^{2}$ indique que le son est émis avec davantage d'énergie, et environ deux tons et demi plus haut. L'écrire $(.)^{2} \mathrm{X}$ indique que le son est deux 
tons et demi au-dessus de la tonalité normale de la voix humaine et qu'il est répété une fois.

Je n'infligerai pas au lecteur l'assommant détail du système ici esquissé. Ce bref exposé de ma méthode de transcription des sons animaux suffira à donner une idée des moyens permettant de noter les sons émis par tous les animaux: de la sorte, celui qui étudie la phonétique saura reconnaître immédiatement le caractère du son, même sans être capable de le reproduire par des moyens naturels.

Il n'est pas sans intérêt de décrire le caractère et l'usage de certains sons prononcés par le chimpanzé. Parmi les sons qu'émettent les animaux, le plus fréquent concerne la nourriture. Il mérite donc la plus grande attention. Dans le langage des chimpanzés, ce mot commence avec le son du [y] bref, combiné avec un [h] puissamment expiré. La commissure des lèvres est serrée et la bouche forme une sorte de rond. Ce son n'est pas difficile à imiter, et les singes le comprennent aisément, même quand on le prononce mal. La méthode de transcription exposée ci-dessus permet de le représenter ainsi, (I)' .

Un autre son fréquemment utilisé parmi les chimpanzés est celui qui sert à appeler. L'élément vocalique est un [y] long, légèrement perçant. Il se perd dans un $[w]$ qui s'évanouit de manière distincte. On le note ainsi: (.) (. Le son qui concerne la nourriture est souvent répété deux ou trois fois de suite, mais celui qui sert à appeler l'est rarement, ou alors à de longs intervalles.

L'un des sons est assez doux et musical: il exprime la sympathie ou l'amitié. Il est plus ou moins doux et plus ou moins long à proportion de l'intensité du sentiment. L'élément vocalique est un [y] long. Il se combine avec un $[\mathrm{h}]$ aspiré. On le représente de manière relativement juste avec le symbole (:)'.

Le son le plus complexe que j'aie entendu est celui que je décris ailleurs comme signifiant «bon ». Les chimpanzés l'utilisent un peu comme font les hommes lorsqu' ils disent « merci », ou « je vous remercie ». Les singes ne l'utilisent probablement pas comme une marque de politesse, mais ce son exprime la même idée.

L'un des signaux d'avertissement ou d'alerte contient un élément vocalique qui ressemble au [ø] bref. Il se termine avec le son du [h] expiré. Il sert à annoncer que quelque chose de familier approche, quelque chose dont l'animal n'a pas peur. S'il s'agit d'un ennemi, ou de quelque chose d'étrange, le même élément vocalique est utilisé, mais est terminé par un 
[h] aspiré distinctement et avec énergie. L'élément vocalique est le même dans les deux mots, mais le temps nécessaire pour les prononcer diffère, ainsi que le fait d'aspirer ou d'expirer à la fin. Il existe aussi une différence dans la manière dont l'émetteur profère le mot. Elle montre clairement qu'il connaît l'usage et la valeur des sons. À l'approche d'un danger, la fin du mot est souvent soufflée dans un murmure, et le mot repris à de longs intervalles, de plus en plus fort à mesure qu'approche le danger. L'autre mot est d'ordinaire prononcé distinctement, et répété plusieurs fois. Il est utile de noter que les Indiens utilisent un mot similaire de la même manière et dans le même objectif.

D'autres sons sont faciles à identifier, mais difficiles à décrire, comme ceux qui signifient le «froid» ou la «sensation désagréable», un autre pour «boire » ou «soif », un autre qui renvoie à l'idée de «maladie», et un autre encore dont j'ai des raisons de croire qu'il désigne l'adjectif « mort » ou « la mort ». Il existe peut-être une douzaine d'autres mots qui sont faciles à identifier, mais dont je n'ai pas encore réussi à déterminer la signification exacte. J'ai ma petite idée sur certains, sans pour autant être encore parvenu à une conclusion définitive.

Le chimpanzé utilise un petit nombre de signes qui peuvent être considérés comme des éléments destinés à appuyer son expression. En signe de négation, il secoue la tête d'un côté et de l'autre, de la même manière que l'homme, sauf qu'il effectue ce geste de manière moins fréquente et moins marquée. Un autre signe de négation, plus commun, consiste à effectuer avec la main un mouvement de balai depuis le corps vers la personne ou la chose destinataire. Ce geste est souvent effectué avec beaucoup d'emphase. Sa signification ne laisse aucun doute, quoique la façon de le faire ne soit pas toujours identique. Quelquefois, c'est un mouvement rapide de la main. Il consiste à envoyer le dos de la main vers la personne ou la chose qui s'approche, en partant du côté opposé. Il signifie que le singe est opposé à cette approche. Le singe pourra effectuer le même signe pour refuser quelque chose qu'on lui présente. Ce geste peut également être fait avec le bras tendu en avant, la main pendante, et son dos tourné vers celui qui approche ou vers la chose refusée. Outre ces signes de négation, il s'en trouve un qui peut être considéré comme un signe d'affirmation. Il consiste simplement à tendre le bras en direction de la personne ou de la chose désirée. Il sert parfois à saluer. Dans ce geste, la main ne bouge pas. Ces signes paraissent innés et sont très proches de ceux qu'utilisent les hommes pour exprimer les mêmes idées. 
Ne concluons pas de cette petite liste de mots et de gestes qu'il n'y aurait plus rien à découvrir. L'étude de la parole des grands singes n'en est pour ainsi dire qu'à ses premiers pas. Plus leurs sons nous deviennent familiers, plus nos difficultés à les comprendre diminuent. Mes espoirs d'apprendre quelque chose de ces animaux n'ont pas été déçus. Le nombre total de mots que je suis désormais capable de distinguer tourne autour d'une centaine. J'ai réussi à interpréter une trentaine d'entre eux.

Je n'ai pas accordé d'attention aux petits singes dans ce chapitre. Je résumerai un jour ce que j'ai pu étudier chez eux, car cela constitue une part essentielle du travail que je me suis donné. Le fait que les animaux soient capables d'interpréter la parole humaine est en lui-même la preuve qu'ils ont l'instinct de la parole. Mais une étude attentive de leurs comportements confirme largement qu'ils possèdent la faculté de parler, et qu'ils s'en servent. Sans compter qu'il leur arrive d'enrichir leur langage avec de nouveaux sons. Cela s'appelle le progrès. Si un grand singe peut faire un pas en avant en matière de langage, pourquoi ne pourrait-il pas en faire deux ? L'exemple que j'ai cité dans le chapitre portant sur Moïse, mon compagnon singe, est à mes yeux la plus belle réussite à laquelle je sois parvenu dans mes efforts pour étudier et former les singes: le fait que j'aie réussi à lui enseigner un mot du langage humain. À lui seul, il est la démonstration que l'animal est doté des ressources de la parole.

Pour conclure, je veux l'affirmer une nouvelle fois : les sons prononcés par les grands singes possèdent les caractéristiques de la parole humaine. Celui qui s'exprime connaît la signification du son qu'il utilise. Il règle la hauteur et le volume de sa voix en fonction de la situation. Le grand singe sait que le son a le pouvoir de transmettre une pensée. Cela, et bien d'autres éléments encore, montrent que les sons qu'il profère appartiennent véritablement au domaine de la parole.

Comparer les facultés mentales des singes sauvages avec celles des chiens domestiques ne permet pas de mesurer avec justice leurs capacités respectives. Le chien a beaucoup appris de sa longue intimité avec l'homme. Si l'on domestiquait le singe, et aussi longtemps que le chien l'a été, il serait plus clairvoyant que le chien, de même qu'il l'est naturellement par rapport aux représentants sauvages de la race canine.

Traduit de l'anglais (États-Unis) par Grégoire Leménager et Laurence Marie. 